\title{
Electra IRP voltage control strategy for enhancing power system stability in future grid architectures
}

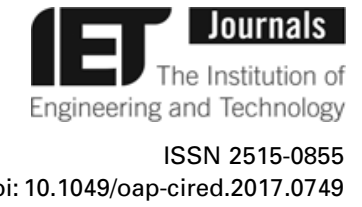

doi: 10.1049/oap-cired.2017.0749

www.ietdl.org

\author{
Julia Merino ${ }^{1}$, J.E. Rodríguez-Seco ${ }^{1}$, Íñigo García-Villalba², \\ Armağan Temiz ${ }^{3}$, Chris Caerts ${ }^{4}$, Roman Schwalbe ${ }^{5}$, \\ Thomas I. Strasser ${ }^{5}$ \\ ${ }^{1}$ Energy and Environment Division, TECNALIA, Derio, Spain \\ ${ }^{2}$ Electrical Engineering Department, University of the Basque Country, Bilbao, Spain \\ ${ }^{3}$ TUBITAK MAM, Ankara, Turkey \\ ${ }^{4}$ VITO, Mol, Belgium \\ ${ }^{5}$ AlT, Vienna, Austria \\ $\bowtie$ E-mail: julia.merino@technalia.com
}

\begin{abstract}
This study is intended to show the future voltage control strategy designed in the framework of the ELECTRA Integrated Research Programme (IRP) project for the web-of-cells (WoC) concept. This scheme, called post-primary voltage control, aims to keep the node voltages at any time within the bands defined by the regulations and to minimise the power losses in the system by calculating the optimal voltage set-points for the different nodes. The reactive power will mainly be used to restore the voltages but also active power could be delivered in some cells depending on their voltage levels. Different operating modes of the voltage control will be discussed taking both planning and the real-time operation phases into account. The application of the voltage control strategy to one test grid considered as representative of the WoC structure will also be shown in this study.
\end{abstract}

\section{Introduction}

The increase in the number of distributed energy resources (DERs) in the power network is driving to significant changes in distribution grids. A variety of new emerging actors, such as energy storage devices, demand-response management and the improvement of power electronic and control devices are providing new options for the provision of electricity to the customers. In this context, the ELECTRA IRP project [1] is working on the definition of new grid topologies that will be representative of the $2030+$ power network as well as in the definition of the associated balancing/frequency and voltage control schemes. This novel grid architecture, called web-of-cells (WoC) [2], proposes a de-centralised scheme for both voltage and balancing/frequency control. The cell, according to definition of the concept developed in the project, is self-sufficient in terms of reserves for solving the voltage and frequency issues in real-time operation and is under the supervision of a cell system operator (CSO). The coordination between neighbouring cells allows the import/export of structural power flows to ensure system security and stability, but not for real-time operation, where the focus in ELECTRA IRP is placed. Only some exceptions are considered for the frequency control cases.

In a power system, the voltage control has to be designed to provide sufficient reactive power to maintain the voltage levels within defined bands in normal operation state. The system operators (SOs) are responsible of performing the voltage control in their own grid. Nowadays, these mechanisms for the voltage control support are uneven for different European countries in both the control structure and the associated remuneration schemas. However, it can be concluded that the SOs are making an effort to go to more complex hierarchical schemes to address the challenges for the future [3]. The massive installation of DERs in distribution grids will have an impact on the power quality parameters, leading to an increase in the voltage variations/fluctuations [4]

\section{The proposed post-primary voltage control (PPVC) scheme}

For avoiding voltage instabilities in power distribution grids, a combination of preventive and corrective approaches has to be combined to obtain an integrated voltage control schema. In case of unexpected events, the time is critical and limited to implement the corrective actions, thus the amount of required reserves has to be guaranteed in the previous period. In this way, the CSO will have a sufficient time to react and take the required decisions. In the voltage control schema defined in ELECTRA IRP, named PPVC, the window-ahead planning actions are included in the so-called 'proactive' mode while the real-time response to big disturbances is handled by the 'corrective' mode.

The goal of the PPVC is to determine optimal voltage set-points for nodes equipped with automatic voltage regulation (AVR) functionality [to perform the primary voltage control (PVC)] and status/position for nodes with discrete control, like transformers with on-load tap changers, capacitor banks, shifting transformers or interruptible loads. The optimality in the system is determined by an optimal power flow (OPF) algorithm such as an interior-point method (IPM) or a genetic algorithm, which calculates the optimal voltage set-points that ensure power flow losses minimisation. The objective to be fulfilled by the voltage control scheme is twofold:

- Calculate voltage set-points that are within the safe band specified by the regulation considering a tolerance around the band limits to avoid the recalculation of the set-points too often, as shown in (1):

$$
V_{\text {sb,min limit }}-\Delta V<V<V_{\text {sb,max limit }}+\Delta V
$$

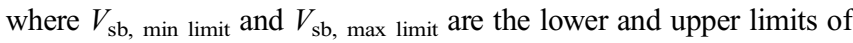
the safe band, respectively, and $\Delta V$ is an additional margin to those limits, to avoid excessive number of OPF processes. 
- Calculate the voltage set-points that optimise the power flows for getting minimum losses (2):

$$
\operatorname{MIN} P_{\mathrm{L}}=\frac{1}{2} \sum_{i=1}^{N} \sum_{\substack{j=1 \\ j \neq 1}}^{N} g_{i, j}\left[V_{i}^{2}+V_{j}^{2}-2 V_{i} V_{j} \cos \left(\delta_{i}-\delta_{j}\right)\right]
$$

where $P_{\mathrm{L}}$ are the total losses in transmission lines, $N$ is the number of nodes, $g_{i j}$ is the conductance between nodes $i$ and $j$, and $V_{i}$ and $V_{j}$ are the voltages in nodes $i$ and $j$ with associated angles $\delta_{i}$ and $\delta_{i}$.

The voltage status detection at the nodes is carried out by the measurement units at the nodes (either active, with AVR, or passive, without AVR) in real-time. The measurements are sent to the cell controller via a reliable cell observing structure. The activation for the 'corrective' PPVC (i.e. OPF is launched and new set-points are calculated) is performed by the cell controller (i.e. PPVC controlling function) if the voltage in any node is out-of-safe band and the PVC controls automatically have entered into operation. Whatever is the node voltage (i.e. even when there is no voltage incident), the operation of the voltage control is done automatically and periodically in every $15 \mathrm{~min}$ ('proactive' PPVC). This time window has been selected with demonstrative purposes. The period length can be variable according to the system characteristics (e.g. the periods when the forecasts are available). However, $15 \mathrm{~min}$ represents a time window length that is a trade-off between the computational cost and the accuracy of the forecasts, for which the efficiency is very high [5]. The operation of the resulting PPVC is shown in the timeline in Fig. 1.

In the proactive mode, before the 15 min cycle $\left(t_{A-1}\right)$ starts, the cell operator has received the information of the tie-line power schedules, the generation and load forecasts based on historical data, the wind speed and solar irradiance measurements etc. and has the static information concerning the network topology to execute the OPF algorithm. Once executed the algorithm, the optimal set-point profiles are sent to every node in the grid, according to the sampling ratio of the measurements defined in the forecasts. By way of example, in a first approach it could be considered $1 / 60 \mathrm{~Hz}$ as the sampling ratio of measurements.

If there are no unscheduled events in the network, the sequence would continue without any interruption in consecutive $15 \mathrm{~min}$ cycles. In case the cell monitoring system detects one unexpected voltage out of the safe bands defined by the regulators (in $t_{A^{\prime}}$ ), the pre-set optimal voltage profile will be no longer valid and the CSO interrupts the actual control cycle, recalculates the optimal voltage set-points according to the new conditions and starts the process again $\left(t_{A+1}^{\prime}-t_{A+2}^{\prime}\right)$ to send the new set-points to be reached after the operation of the PVC controls. It can be concluded that

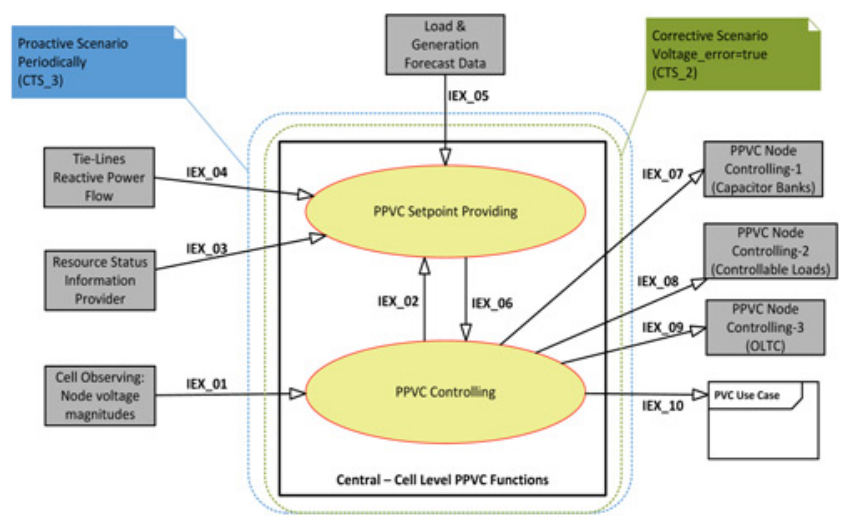

Fig. 2 Context diagram PPVC

the PPVC philosophy is the same whether the system is in undisturbed regime ('proactive' mode) or disturbed regime ('corrective' mode) with the only difference in this last situation the control cycle length is shortened.

Most of the time, the 'proactive' mode of the PPVC will be sufficient for the operation of the WoC in a secure manner. The fluctuations coming from foreseen generation-load imbalances are considered in the operational planning phase, thus the preventive actions have been already implemented. Only in the case of severe unexpected incidents, the 'corrective' mode will begin its operation after the PVC.

The context diagram, in Fig. 2, shows the relationship between the main functions of the PPVC (in yellow) and the needed information exchanges between both functions (IEX_X) as well as the required inputs (grey boxes) [6].

As it can be also observed from Fig. 2, the functions to be integrated into the CSO as the core part of the PPVC scheme are (i) the PPVC set-point providing function, and (ii) the PPVC controlling function. Both are common for the 'proactive' mode (represented with a blue dashed box) and the 'corrective' mode (green dashed box).

The PPVC set-point providing function determines the node set-points using OPF algorithms (i.e. determination of set-points for PVC nodes with AVR capabilities, and PPVC nodes with capacitor banks or transformer tap changers). This function uses reactive power reserves margins (and the location of those reserves), forecasted generation/load data and active/reactive power flows of tie-lines as inputs to determine PVC/PPVC resources set-points to keep the voltage profile within the regulatory limits and meet the optimisation objectives.

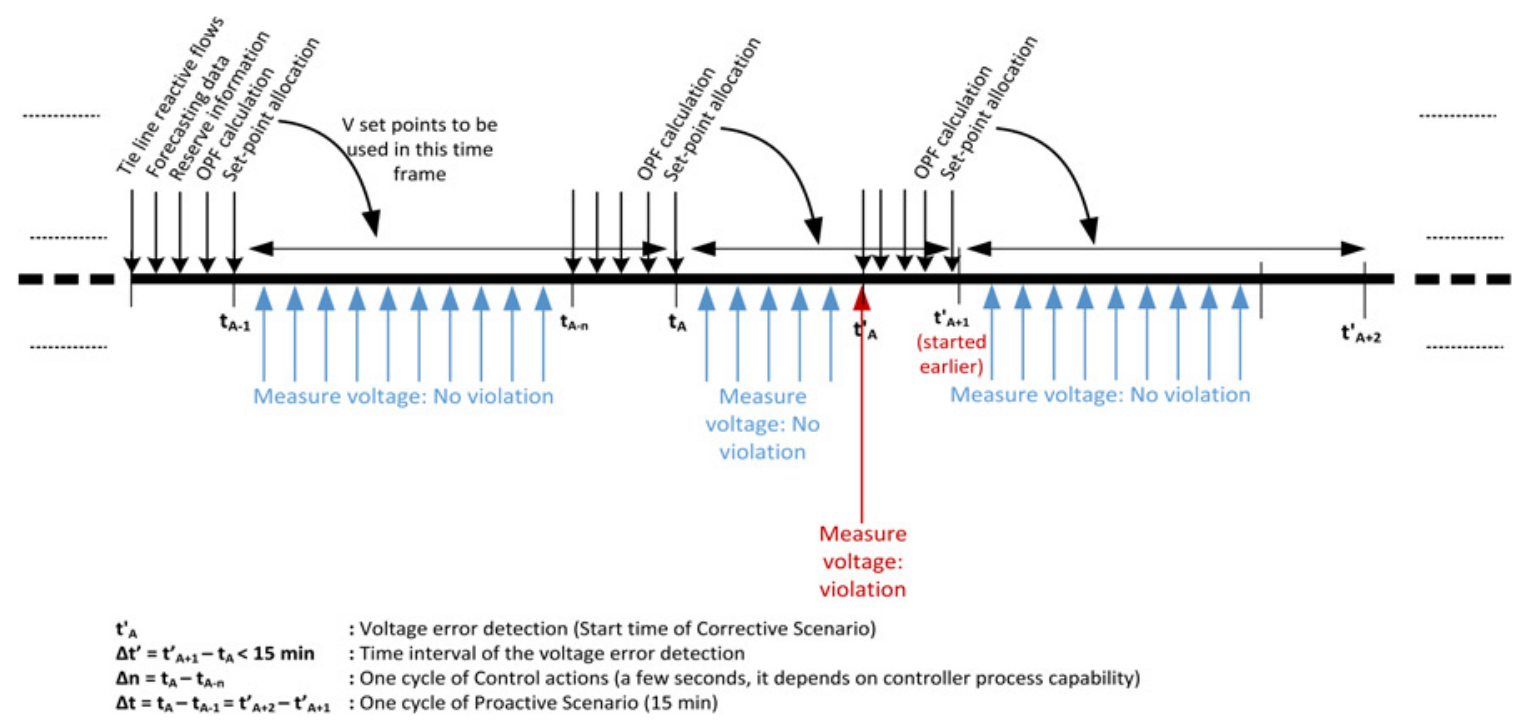

Fig. 1 PPVC timeline 
Table 1 Information exchanges

\begin{tabular}{|c|c|}
\hline Code & Information exchanged \\
\hline IEX_01 & $\begin{array}{l}\text { periodical (e.g. sampling }=1 \mathrm{~Hz}) \text {. Array of voltage measurements } \\
(\mid \mathrm{V} \text {, in } \mathrm{V}) \text { and location information for each cell node }\left[V_{i} \mathrm{EAN}_{i}\right]\end{array}$ \\
\hline IEX_02 & $\begin{array}{c}\text { trigger signal/command (binary value) to perform OPF and get } \\
\text { current IEX_03, IEX_04, and IEX_05 }\end{array}$ \\
\hline IEX_03 & $\begin{array}{l}\text { array of SVC reserve capacity information ( } Q \text { in VAr), availability } \\
(1 \text { or } 0) \text {, and the location of the resource }\left[Q_{i} \text { stati EAN }_{i}\right]\end{array}$ \\
\hline IEX_04 & $\begin{array}{r}\text { array of cell tie-lines reactive powe } \\
\text { information and locat }\end{array}$ \\
\hline IEX_05 & $\begin{array}{l}\text { output of the gene } \\
\text { - array of forecasted generation } \\
\text { the next time window } \\
\text { output of the load } \\
\text { - array of forecasted load value } \\
\text { next time window (e.g }\end{array}$ \\
\hline IEX_06 & 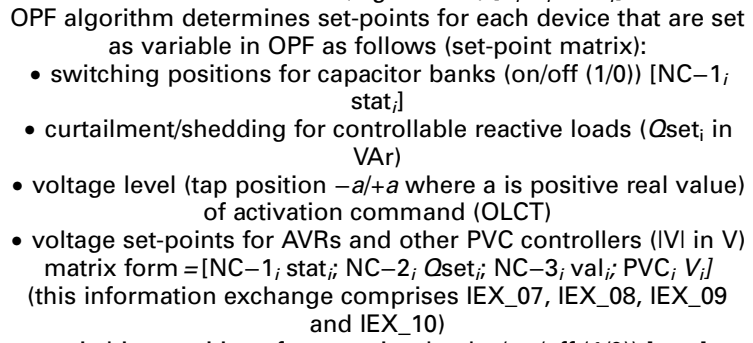 \\
\hline $\begin{array}{l}\text { IEX_07 } \\
\text { IEX_08 }\end{array}$ & $\begin{array}{l}\left.\text { switching positions for capacitor banks (on/off }(1 / 0)) \text { [stat }{ }_{i}\right] \\
\text { curtailment/shedding for controllable reactive loads }\left(Q_{i} \text { in VAr) }\right. \\
\left.\text { [Qset }{ }_{i}\right]\end{array}$ \\
\hline IEX_09 & $\begin{array}{c}\text { voltage level (tap position }-a /+a \text { where } a \text { is positive real value) } \\
\left.\text { of activation command (OLCT) [val }{ }_{i}\right]\end{array}$ \\
\hline IEX_10 & $\begin{array}{l}\text { voltage set-points for AVRs and other PVC controllers (IV/ in V) } \\
\qquad\left[V_{i}\right]\end{array}$ \\
\hline
\end{tabular}

The PPVC controlling function determines whether the node voltages are in the safe-band or not by checking the node voltage values. If a voltage value is out of range, this function sends the voltage error/activation signal to the PPVC set-point providing function to calculate new optimal values. This function uses voltage magnitudes from the cell nodes to compare the node voltage measurements with the safe-band limits (specified by the regulation). It also gets the calculated set-points from PPVC set-point providing function to transform them to a usable format. Eventually, it sends the set-points to the PPVC node controllers and the PVC controllers.

The details of the information flows exchanged between the different functions as well as their input functions are summarised in Table 1.

\section{Case study: the CIGRE-MV grid}

As an example of a WoC test grid to be used within the ELECTRA IRP project, the European CIGRE medium-voltage (MV) benchmark with integrated DER resources has been chosen as a candidate [7]. Its layout is shown in Fig. 3. As the voltage control mechanism operating in stand-alone mode does not require the coordination with neighbouring cells, this grid is going to be considered in this study as a single cell.

The connection to the high-voltage grid is represented through a $110 \mathrm{kV}$ busbar. Downstream the grid has two main MV feeders where the loads and the DERs are connected at $20 \mathrm{kV}$ level. Both feeders are weakly linked. The DERs are mainly photovoltaic (PV) installations on the low-voltage (LV) level (i.e. 8 PV plants), but also batteries ( 2 units), combined-heat power (CHP) devices (2 units) and fuel cells (2 units) are connected at $0.4 \mathrm{kV}$ while the wind turbine is connected at $0.69 \mathrm{kV}$.

According to the available data of rated values present in [7] dedicated PQ load profiles as well as the DERs profiles have been created based on real measurements. By way of example the active power patterns of aggregated PV panels are shown in Fig. 4.

A simulation test (i.e. a scenario) is going to be developed to show the full operation cycle of the PPVC over the CIGRE MV. Before $t_{A-1}(17: 00 \mathrm{~h})$, the cell operator receives the forecasts of the PQ

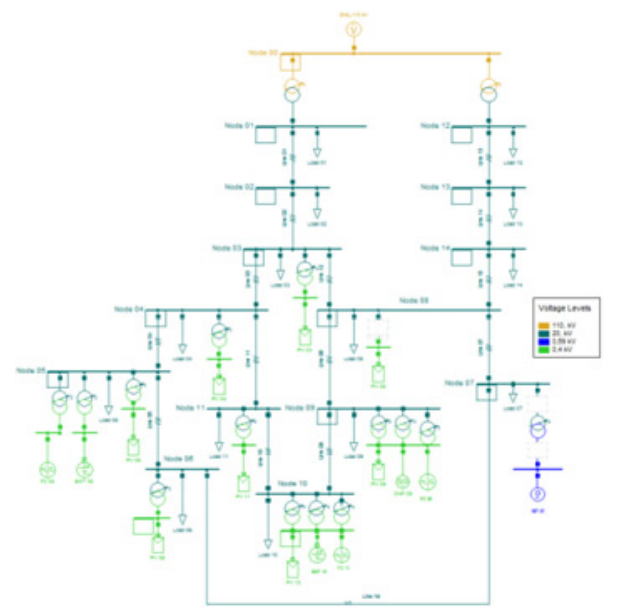

Fig. 3 CIGRÉ $M V$ benchmark topology

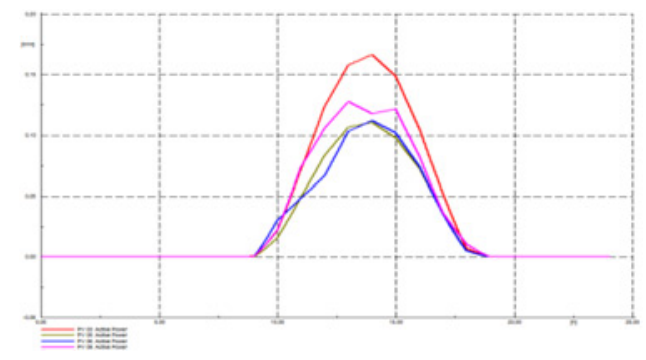

Fig. 4

PV plants generation profiles

loads and the power generation for the next time window (17:0017:15), as shown in Fig. 5 for some representative system loads.

In the operation of the cell, the CSO has to consider the different possibilities available:

(A) (A) Planning phase ('proactive' mode)

(A.1) the execution of the OPF algorithm leads to a feasible situation for the next time window;

(A.2) the execution of the proactive algorithm gives and alarm state due to the non-convergence of the algorithm.

(B) (B) Real-time operation ('corrective' mode).

The execution of the OPF algorithm gives a full feasible optimal voltage profile pattern for the current window but an unexpected event occurs during the real-time cell operation so the previous pattern needs to be modified.

The OPF algorithm optimises the objective function (minimisation of power losses) according to the inequality constraints established: limits of voltage in the nodes, taps in the transformers and active/ reactive power limits of the generators. In the case A.1, the optimised profile of set-points for every node is sent to the different PVC/PPVC controllers for the next period. In the case A.2, the result of the predictive OPF for the future 15 min window leads to a non-convergence state in any moment of the time series, due to a lack of reactive power reserves. The non-convergence of the case for the situation will force the CSO to consider the addition of extra resources to ensure the voltage stability of the cell for that period.

The lower and upper limits of the safe band in this example are 0.95 and $1.05 \mathrm{pu}(5 \%)$, respectively. These limits are orientative because they are highly variable depending on the regulation applicable. Nevertheless, the voltage control scheme is independent of these limits. The voltage control, due to its local nature, is highly dependent on the location of the problem; therefore, it is usual to have dedicated power quality recommendations applied by each TSO/DSO. The European Standard EN-50160 allows voltage 

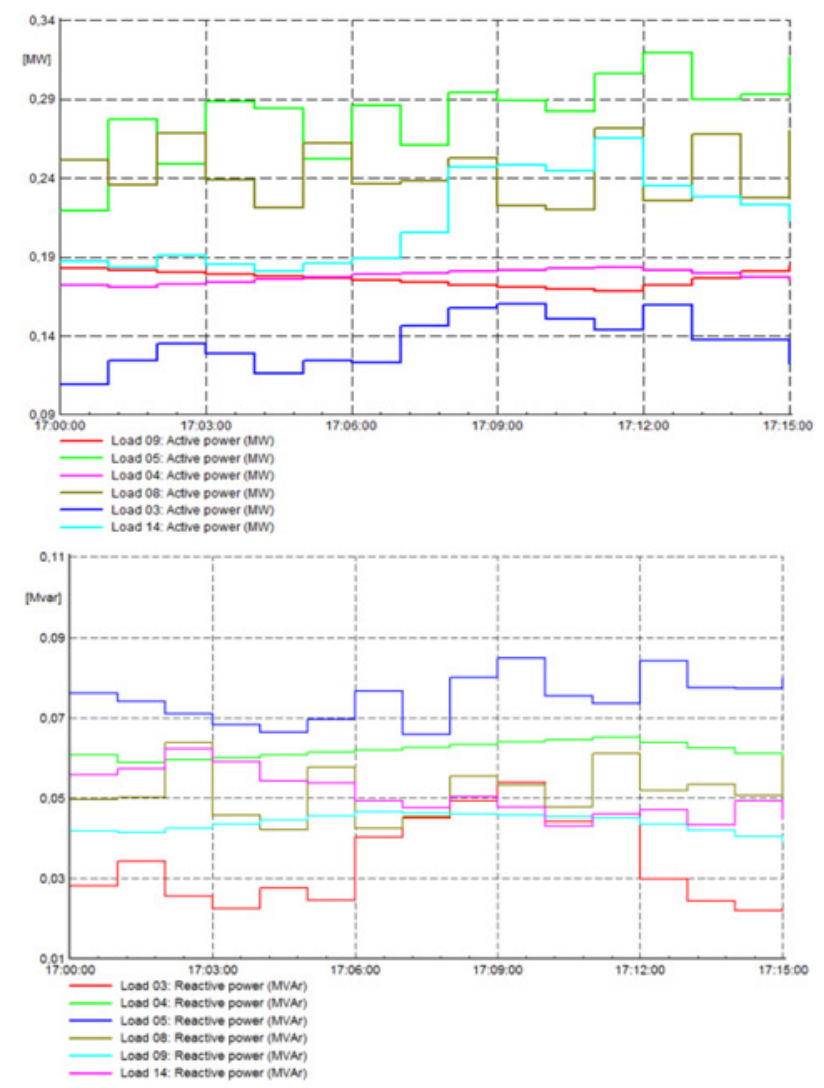

Fig. 5 Active/reactive power load patters

variations in the point of common coupling in distribution grids of $\pm 10 \%$ [8] while the Spanish Regulation is more restrictive, establishing those limits in $\pm 7 \%$ [9].

The simulation of the case A.2, displayed in Fig. 6, indicates that one of the nodes at the end of the first feeder would be out of the safe-band in $t_{A+1}^{\prime}$, what effectively would happen with no proactive regulation in real-time operation (Fig. 7). Then the CSO has to incorporate new resources to the WoC that will increase the reactive power reserves. Due to the anticipation of the voltage problem the CSO can consider the droop $(\mathrm{Q} / \mathrm{U})$ associated to the DERs in the planning phase to activate the resources prior the voltage drop occurrence, reducing the response timings in the WoC.

Fig. 8 shows the results of case (B. The voltage profile has been settled for as a result of the proactive operating mode for a generic time window $t_{A-1}$ and $t_{A-2}$ (10:00-10:15 in the figure). This profile is maintained for the whole period but an unexpected error happens at $t_{\mathrm{A}-1}^{\prime}$. New optimal set-points have to be sent to the devices for a starting window of $15 \mathrm{~min}$ once the proactive operation has guaranteed the cell has enough reserves available for the real-time operation phase (i.e. the voltages can recover up to the safe band values).

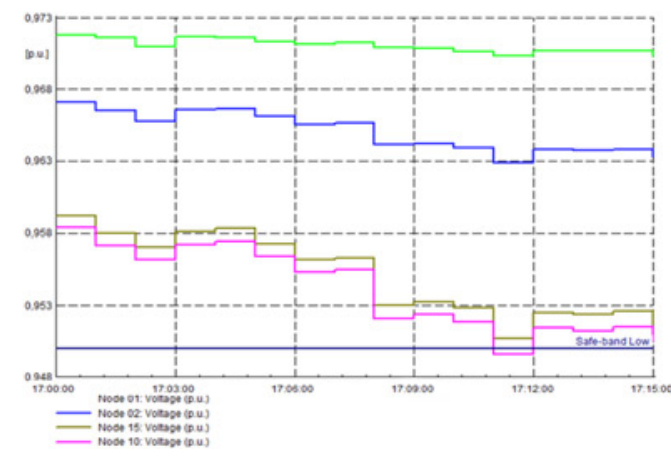

Fig. 6 Expected voltage deviations in the window-ahead period

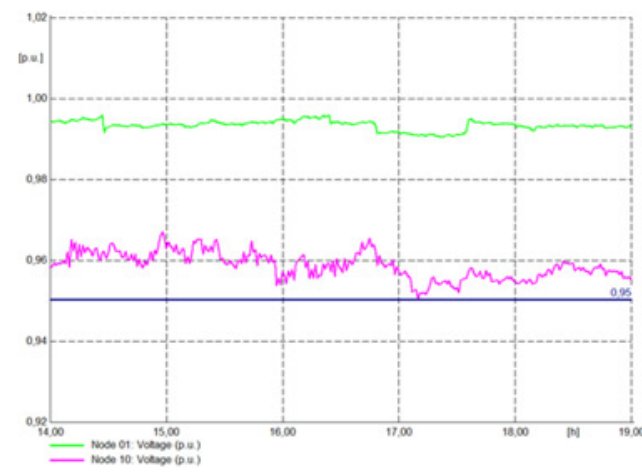

Fig. 7 Voltage deviation in real-time without proactive control

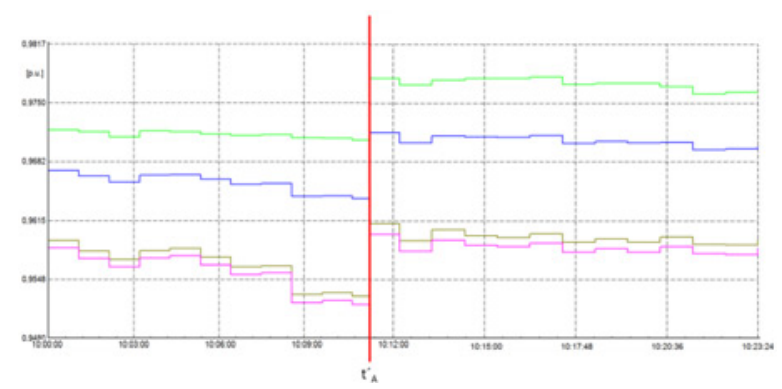

Fig. 8 Corrective mode

This method supposes an evolution over the current voltage control mechanisms currently in use. It gives the optimal voltage set-points to the DER units for the next operation time window. This reduces the complexity of the traditional voltage schemes developed in three steps (primary/secondary/tertiary), improving the system efficiency. In practice, nowadays the implementation of a centralised OPF is still troublesome [3]. However, the PPVC could be possible in the $2030+$ horizon where ELECTRA IRP focuses, thanks to the advances in grid observability and the rise of the calculation capacities, the communication systems and the data processing algorithms that are constitutive of the WoC.

\section{Conclusions}

This paper presents a voltage control structure defined within the ELECTRA IRP project and its application to a cell (i.e. pilot network). The voltage control structure is thought as a holistic approach that considers both the planning phase and the real-time operation. It is rooted on the advantages presented by the WoC concept, where the vast deployment of measurement devices and the wide use of the information and communication technologies will increase the CSO capacities. This will allow a new voltage control scheme, PPVC, to be applied in 2030+ horizon to mitigate prospective grid instabilities.

\section{Acknowledgments}

The research leading to these results has received funding from the European Union Seventh Framework Programme (FP7/20072013) under grant agreement no. 609687.

\section{References}

1 ELECTRA Integrated Research Programme. Available at http://www.electrairp.eu, accessed on 15 January 2017

2 Caerts, C., D'hulst, R., De Breucker, S., et al.: 'Specification of smart grids high level functional architecture for frequency and voltage control'. ELECTRA 
Deliverable D3.1. WP3: Scenarios and Case Studies for Future Power Systems, 2015, Available for download at http:// www.electrairp.eu/, accessed on 15 January 2017

3 Mousavi, O.A., Cherkaoui, R.: 'Literature survey on fundamental issues of voltage and reactive power control' (EPFL and ETH, Lausanne, Switzerland, 2011)

4 Bollen, M., Häger, M.: 'Power quality: interactions between distributed energy resources, the grid, and other customers' (STRI AB, Sweden, 2005)

5 Hodge, B.M.: 'Wind power forecasting error distributions: an international comparison' (NREL, USA, 2012)
6 Caerts, C., Rikos, E., Syed, M., et al.: 'Description of the detailed functional architecture of the frequency and voltage control solution (functional and information layer)'. ELECTRA Deliverable D4.2. WP4, Fully Interoperable Systems, 2016

7 CIGRÉ.Task Force C6.04: 'Benchmark systems for network integration of renewable and distributed energy resources', 2014

8 CENELEC: 'EN50160: voltage characteristics of electricity supplied by public distribution systems', 2001

9 Boletín Oficial del Estado (BOE): 'Real Decreto 1955/2000. Artículo 104', Spain 